\title{
Hydration and Temperature Response of Water Mobility in Poly(diallyldimethylammonium)-Poly(sodium 4-styrenesulfonate) Complexes
}

\author{
Piotr Batys, ${ }^{\S, \perp, \| \odot ~ Y a n p u ~ Z h a n g, ~}{ }^{\dagger}$ Jodie L. Lutkenhaus, ${ }^{*}, \dagger, \ddagger \odot$ and Maria Sammalkorpi ${ }^{*}, \S \odot$
}

${ }^{\S}$ Department of Chemistry and Materials Science and ${ }^{\perp}$ Department of Bioproducts and Biosystems, School of Chemical Engineering, Aalto University, P.O. Box 16100, FI-00076 Aalto, Finland

"Jerzy Haber Institute of Catalysis and Surface Chemistry, Polish Academy of Sciences, Niezapominajek 8, PL-30239 Krakow, Poland

${ }^{\dagger}$ Artie McFerrin Department of Chemical Engineering and ${ }^{\ddagger}$ Department of Materials Science and Engineering, Texas A\&M University, College Station, Texas 77843, United States

\section{Supporting Information}

ABSTRACT: The combination of all-atom molecular dynamics simulations with differential scanning calorimetry (DSC) has been exploited to investigate the influence of temperature and hydration on the water distribution and mobility in poly(diallyldimethylammonium) (PDADMA) and poly(sodium 4-styrenesulfonate) (PSS) complexes. The findings show that the vast majority of the water molecules hydrating the polyelectrolyte complexes (PECs) with 18-30 wt \% hydration are effectively immobilized due to the strong interactions between the PE charge groups and water. Temperature and hydration were found to decrease similarly the fraction of
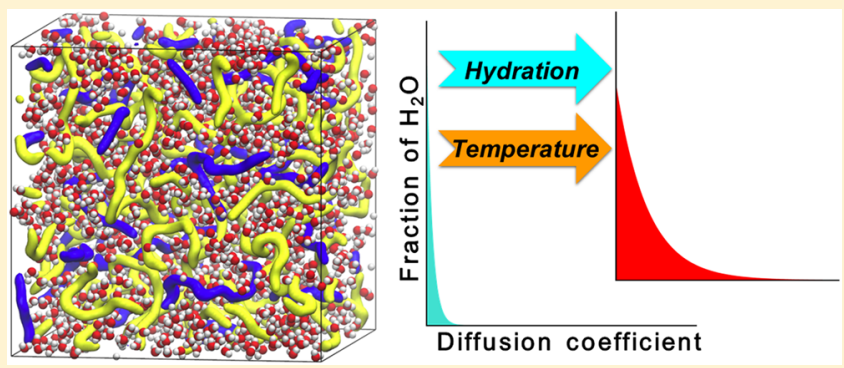
strongly bound water. Additionally, at low hydration or at low temperatures, water motions become dominantly local vibrations and rotations instead of translational motion; translation dominance is recovered in a similar fashion by increase of both temperature and hydration. DSC experiments corroborate the simulation findings by showing that nonfreezing, bound water dominates in hydrated PECs at comparable hydrations. Our results raise attention to water as an equal variable to temperature in the design and engineering of stimuli-responsive polyelectrolyte materials and provide mechanistic explanation for the similarity.

\section{INTRODUCTION}

Simple mixing of oppositely charged polyelectrolytes (PEs) in aqueous solutions due to entropy driven phase separation, ${ }^{1}$ leads to the formation of polyelectrolyte complexes (PECs). The relatively easy preparation method in comparison to polyelectrolyte multilayers (PEMs) has contributed to the wide use of PECs and PEC-based materials in industrial applications, for example, as flocculants for colloidal dispersion, adhesives, water purification, and hydrophilic soil binders. ${ }^{2}$ Additionally, synthesis of PECs does not require the use of a chemical crosslinking agent which reduces toxicity and makes many PECs promising materials in the fields of biotechnology and medicine, for example, for encapsulation of drugs and enzymes, for immobilization and purification of proteins, ${ }^{5}$ and as vectors in gene therapy. ${ }^{6}$

The properties of the PECs can be controlled either by varying formation conditions, such as $\mathrm{pH}$ or ionic strength, as well as PE chemistry and concentration, ${ }^{7,8}$ or by postproduction modification, such as drying or hydration, ${ }^{9}$ thermal annealing, ${ }^{10}$ ion-exchange, ${ }^{11,12}$ or surface functionalization. ${ }^{13}$ Their formation mechanisms, ${ }^{14-16}$ as well as physicochemical properties including conductivity ${ }^{17,18}$ and mechanical ${ }^{19}$ and thermal properties, ${ }^{19,20}$ have been intensively studied. Of these, the thermal properties are particularly interesting as hydrated PECs undergo a glass-transition-like thermal transition. ${ }^{7,11,20-24}$ At a specific temperature PECs become softer. ${ }^{25}$ The transition response and ensuing plasticization provides a handle to control the PEC mechanical properties ${ }^{21,22}$ and allows them to be used as thermally responsive materials. ${ }^{26}$ On the other hand, water has a crucial role as a plasticizer in this phenomenon: dried PECs are brittle and thermally stable and exhibit no thermal transitions even at elevated temperatures. ${ }^{20}$ The transition temperature of hydrated PECs has been reported to decrease with increasing water content and salt doping level. ${ }^{11,21,25}$ Additionally, recent studies ${ }^{21,22}$ have connected the thermal transition with shortening of $\mathrm{PE}$-water hydrogen-bonding lifetime, which facilitates PE motions. The mobility of PE chains is additionally enhanced by water through providing

Received: July 6, 2018

Revised: September 25, 2018

Published: October 11, 2018 
volume for PE chain motion and reducing the friction between them. ${ }^{27-29}$ On the other hand, fully hydrated PEMs might not exhibit transition in the accessible temperature range. ${ }^{30}$ Even though the plasticizing effect of water in hydrated PECs has been generally described, further and more detailed explanations of PEC response to water and the underlying mechanism are needed. ${ }^{19}$ Specifically, an understanding of the state of water (bound vs nonbound) and its diffusion (rotational and translational) in the PEC is needed.

In PECs, water is confined by the PECs' hierarchical microand nanoporous structure; such confined geometries have been reported to result in water exhibiting surface-dependent properties, anomalous diffusion, ${ }^{31,32}$ evaporation, ${ }^{33}$ or melting or freezing temperature ${ }^{34-36}$ responses. The responses depend on degree of confinement, that is, pore size and changes in pore sizes. For PEC aggregates, pore sizes on the order of tens of micrometers have been reported while smaller nanopores are generated upon the PE complexation, that is, the PEC is a kinetically trapped PE network that rearranges very slowly. ${ }^{37}$ Porosity in hydrated PECs can be decreased, and the PEC can be compacted by dehydration, mechanical pressing, or centrifugation $^{38}$ and regenerated by soaking in salt solution over a long period of time. ${ }^{37}$ For the poly(diallyldimethylammonium) (PDADMA)/poly(sodium 4-styrenesulfonate) (PSS) PEM, experimentally determined pore sizes of about $1 \mathrm{~nm}$ have been reported. ${ }^{39}$

Water in hydrated PECs may be considered to exist in three different states based on its melting temperature $T_{\mathrm{m}}$, as measured using differential scanning calorimetry (DSC): (1) nonfreezing, bound water (no detectable $T_{\mathrm{m}}$ ), (2) freezing, bound water $\left(T_{\mathrm{m}}\right.$ below $\left.273 \mathrm{~K}\right)$, and (3) freezing, relatively free water $\left(T_{\mathrm{m}} \sim 273 \mathrm{~K}\right){ }^{40}$ The depression in $T_{\mathrm{m}}$ for bound water may be related to either weaker interactions of water with the PEs, for example, formation of a second hydration shell, or with the porous structure of the PEC. Water that can be frozen, that is, freezing bound water or freezing relatively free water, is hypothesized to not be present at low PEC hydration. On the other hand, the polyelectrolyte ion pairs are hydrated with water so tightly bound that it does not experience freezing: this nonfreezing bound water may provide the major contribution to the plasticization of hydrated polymers. ${ }^{27}$ Furthermore, Hodge et al. ${ }^{27}$ have shown that, for swollen poly(vinyl alcohol), a critical water content (in that case $30 \mathrm{wt} \%$ ) is required for freezing water to be present; at lower hydrations, all water is so tightly bound that it does not freeze. The critical water content there corresponds to the theoretical value for complete hydration saturation of the poly(vinyl alcohol) hydroxyl sites. The finding suggests that the critical hydration wt $\%$ at which the $\mathrm{PE}$ system contains sufficiently loosely bound water for it to be able to freeze may depend on the polymer side chain chemistry and size.

Computer modeling, more precisely molecular dynamics $(\mathrm{MD})^{21,22,41-43}$ and Monte Carlo ${ }^{44-47}$ methods, provides one of the most perspective theoretical tools to examine the response of water in confined geometries. $\mathrm{MD}$ simulations have been used to understand PE adsorption ${ }^{48}$ and PEM and PEC formation. ${ }^{42,43,49}$ Coarse grained approaches provide access to PE dynamics in the assemblies. ${ }^{50}$ However, to capture the dynamics of the water and ions in PECs, explicit solvent all-atom MD simulations are used. ${ }^{21,22,24,41,51,52}$ For example, the diffusion of water molecules inside PDADMA/ PSS PECs has been shown to be significantly slower than that in bulk water. ${ }^{52}$ Additionally, simulations have contributed to associating water mobility in PE assemblies with the mechanism of PEC thermal transition, ${ }^{22,24}$ as well as probed the role of water and salt in PEC plasticization. ${ }^{21}$

In this work, we have characterized the water mobility in hydrated PDADMA/PSS PECs via all-atom detail MD simulations and differential scanning calorimetry (DSC). In particular, we have mapped the hydration and temperature response of the water motions both by MD simulations and DSC. The simulations enable assessing the corresponding distribution of water in the PECs. Finally, the findings are connected by modulated differential scanning calorimetry (MDSC) measurements to the glass transition temperature $T_{\mathrm{g}}$. The significance of the work is that it connects the temperature and hydration induced plasticization response of PEC materials and shows they result in similar, interconnected response. This means that water can be considered as a variable similar to temperature in tuning PEC materials characteristics.

\section{MATERIALS AND METHODS}

Experiments. Poly(diallyldimethylammonium chloride) (PDADMAC, 20 wt $\%$ in water, Sigma-Aldrich, $M_{w}=200000-$ $350000 \mathrm{~g} \mathrm{~mol}^{-1}$ ) and poly(sodium 4-styrenesulfonate) (PSS, Scientific Polymer Products, $M_{\mathrm{w}}=500000 \mathrm{~g} \mathrm{~mol}^{-1}$ ) were used as received. PE solutions were prepared using deionized water with $18.2 \mathrm{M} \Omega \cdot \mathrm{cm}$ resistivity. The PECs were obtained by mixing stoichiometric amounts of PDADMAC and PSS solutions under stirring, without any salt addition. The precipitate PECs were centrifuged, pressed, and rinsed with water, and after being dried they were ground into fine powders. Details of PEC preparation and composition estimation are described elsewhere. ${ }^{24}$

The state of water in the hydrated PECs was examined by distinguishing between the freezing and nonfreezing states of water using DSC in standard mode. The sample was first cooled from 313 to $223 \mathrm{~K}$ at $5 \mathrm{~K} \mathrm{~min}^{-1}$, kept isothermal at $223 \mathrm{~K}$ for $10 \mathrm{~min}$, and heated up to $293 \mathrm{~K}$ at the same rate. The endothermic melting peak was analyzed to determine the frozen state water. ${ }^{40}$ Assuming melting enthalpies for both freezing free water and freezing bound water to be the same as that of bulk water, ${ }^{40}$ the amount of freezing water $\left(W_{\mathrm{f}}\right)$ is calculated as

$$
W_{\mathrm{f}}=\frac{\Delta H_{\mathrm{m}}}{\Delta H_{0}}
$$

where $\Delta H_{\mathrm{m}}$ is the observed melting enthalpy of water in the hydrated PEC and $\Delta H_{0}$ is the melting enthalpy of pure water in a similar environment (i.e., sealed in DSC pan). Here, pure water exhibited a melting peak with $329 \mathrm{~J} \mathrm{~g}^{-1}$ fusion heat at $273 \mathrm{~K}$, close to the previously reported value $334 \mathrm{~J} \mathrm{~g}^{-1} .^{40}$ The amount of nonfreezing water $W_{\mathrm{nf}}$ is obtained from difference of total water content and the freezing water $W_{\mathrm{f}}$ as

$$
W_{\text {nf }}=W_{\mathrm{c}}-W_{\mathrm{f}}
$$

Molecular Dynamics Simulation. The Gromacs 5.1.3 package ${ }^{53,54}$ was used for all-atom molecular dynamics (MD) simulations of 23PDADMA $\mathrm{P}_{20}-21 \mathrm{PSS}_{20}$ assemblies (PECs), where the subscript refers to the number of repeat unit in each chain. The prefactors 23 and 21 refer to the number of 20 repeat unit PDADMA and PSS molecules; the PDADMA-to-PSS ratio follows from experimental neutron activation analysis (NAA). The chemical structures of PEs and sample chain conformations are presented in Figure 1. To describe the PEs and the ions, the OPLS-aa force field ${ }^{55}$ with the ammonium groups extension ${ }^{56}$ was used. The partial charges of PSS were taken from ref 52. The parameters for sodium and chloride ions originate from refs 57 and 58, respectively. For water, in compliance with the force-field choice, the explicit TIP4P water model ${ }^{59}$ was employed.

The PME method was used for the long-range electrostatic interactions. ${ }^{60}$ van der Waals interactions were described using the LennardJones potential with a $1.0 \mathrm{~nm}$ cutoff. Throughout the simulations, all 
a)<smiles>CC(C)c1ccc(S(=O)(=O)[O-])cc1</smiles>

b)

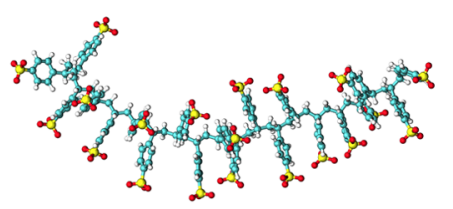

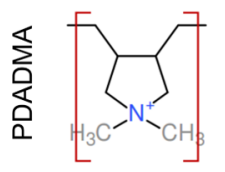
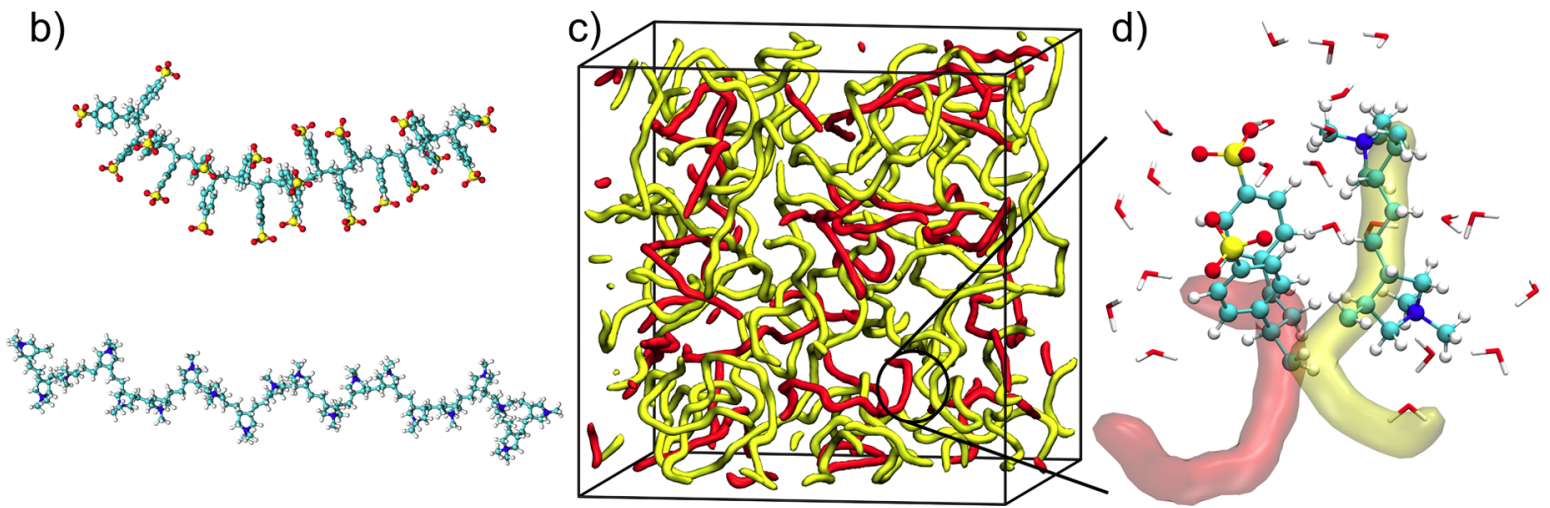

Figure 1. (a) PSS and PDADMA chemical structures and (b) 20 repeat unit long chains from MD simulations. (c) Visualization of the PE backbones in a relaxed initial configuration of $23 \mathrm{PDADMA}_{20}-21 \mathrm{PSS}_{20}$ at $18 \mathrm{wt} \%$ water. For clarity and better visualization of the chain entanglement, the simulation box visualization presents just the PE backbones. (d) An expanded view of a single intrinsic ion pair surrounded by water molecules. The expanded view shows how the PE side chains and water molecules fill the voids. PDADMA backbone is in yellow and PSS in red.

the bonds in the PEs and water molecules were controlled by the LINCS $^{61}$ and SETTLE ${ }^{62}$ algorithms, respectively. A 2 fs (initially $0.5 \mathrm{fs}$ ) time step within the leapfrog integration scheme was applied, and the trajectories were written every 1000 steps. Periodic boundary conditions were applied in all directions. All simulation visualizations were done using the VMD software package. ${ }^{63}$ Temperature was controlled via the $\mathrm{V}$-rescale thermostat ${ }^{64}$ with coupling constant $0.1 \mathrm{ps}$ at reference temperature $290 \mathrm{~K}$. The PEs were coupled to the heat bath as one thermostatting group while water and the ions were coupled to the heat bath as another group. The pressure was controlled via the Parrinello-Rahman barostat ${ }^{65}$ with the coupling constant 2 ps and reference pressure 1 bar.

The initial configurations were generated using PACKMOL, ${ }^{66}$ with PE chain conformations extracted from dilute solution. The detailed steps of obtaining PE assemblies with relatively uniform structure and water distribution are presented in Table S1 in the Supporting Information (SI). ${ }^{24}$ The generated initial configurations were simulated for $100 \mathrm{~ns}$ at an elevated temperature of $370 \mathrm{~K}$, after which the temperature was brought down to $290 \mathrm{~K}$ in $10 \mathrm{~ns}$ time as the relaxation before the production run. See Figure 1 for an example of the degree of PE entanglement after relaxation. In the production run, the temperature was increased from 290 to $360 \mathrm{~K}$ in $5 \mathrm{~K}$ steps of $15 \mathrm{~ns}$ each for a total simulation duration of $225 \mathrm{~ns}$. The first $1 \mathrm{~ns}$ of each step was disregarded in the analysis. The employed time scale is too short to capture polymer chain relaxation and diffusion, but water and ion dynamics are captured. ${ }^{67}$ Temperatures approaching the freezing temperature were not probed by simulations as the accuracy of the simulation results decreases near phase transition points.

For setting up the PECs to match the experimental compositions, we used experimental compositions determined in an earlier work using NAA. ${ }^{24}$ In the simulations, four different water contents, $W_{c}$, similar to the DSC experiments, were studied, that is, $18 \mathrm{wt} \%\left(N_{\mathrm{W}}=\right.$ $1664), 22 \mathrm{wt} \%\left(N_{\mathrm{W}}=2138\right), 26 \mathrm{wt} \%\left(N_{\mathrm{W}}=2664\right)$, and $30 \mathrm{wt} \%$ $\left(N_{W}=3250\right)$ water. Here, $N_{W}$ is the number of water molecules in the simulation box. Our selected hydration range mimics cases in which polyelectrolyte complexes and multilayers are used as solids in humid conditions ${ }^{18,68,69}$ or in the case of studying the glass transition. ${ }^{21-25}$ Higher hydration levels, experimentally observed for fully immersed complexes and multilayers, ${ }^{70}$ were not examined.

To neutralize the system, 40 chloride ions were added to the system. This results in a 1.04 wt $\%$ of $\mathrm{Cl}^{-}$in a dry complex, in close match with the NAA analysis result, that is, $0.91 \mathrm{wt} \%$. The PSS to PDADMA ratio (based on repeat unit molar ratio) in each simulation was 0.913 (0.922 in NAA experiments). All presented results are calculated as an average over simulations with three different initial configurations, unless otherwise stated.

Solvent accessible surface area was calculated using the double cubic lattice method ${ }^{71}$ (Gromacs gmx sasa program) with probe radius $0.14 \mathrm{~nm}$. The calculation was done as an average over the frames of each temperature step. The rotational and translational diffusion coefficients of water were estimated using the two phase thermodynamic model (2PT). ${ }^{72-74}$ To extract the velocity data for this calculation, separate simulations of $50 \mathrm{ps}$ in duration with an enhanced output frequency of 2 fs were run for each system and for each temperature step. The final frame (coordinates and velocities) at each temperature in the temperature ramping was used as the initial configuration of this run. Similarly, to determine the diffusion coefficients for the single water molecules, 20 ps separate simulations with an enhanced output frequency of $10 \mathrm{fs}$ were run for each system at 290,325 , and $360 \mathrm{~K}$ temperature steps. The diffusion results were checked against 10 and 50 ps long simulations; the 20 ps simulations were long enough to lead to the same average and no visible differences, as the 50 ps simulations.

\section{RESULTS AND DISCUSSION}

States of Frozen Water in and the Glass Transition Temperatures of PECs. Standard DSC was used to explore the state of water in the hydrated PECs as compared to pure water. Figure S1 (SI) presents the heating curves of the hydrated samples and the pure water as a control. It should be noted that values determined using DSC are extracted at or near water's freezing point such that the obtained information corresponds only to temperatures in that range. At higher temperatures, the distribution of water may differ significantly, and especially the ratio between the freezing and nonfreezing bound water may change. First, the thermogram of pure water exhibits a considerable endothermic peak with enthalpy of $329 \mathrm{~J}^{-1}$, which is slightly lower than the reported value of $334 \mathrm{~J} \cdot \mathrm{g}^{-1}$ due to the sealed environment. ${ }^{40}$ Second, Figure $2 \mathrm{a}$ shows that only the sample with $W_{\mathrm{c}}=30 \mathrm{wt} \%$ has a small melting peak at temperature well below $273 \mathrm{~K}$, attributed to freezing bound water. The lower hydration levels bore no sign of melting, indicating that only nonfreezing bound water was present. For $30 \mathrm{wt} \%$ hydration, the amount of freezing bound water was calculated from the integration of the melting peak to be $0.9 \mathrm{wt} \%$ of the hydrated PEC. The melting peak is relatively wide, from 255 to $270 \mathrm{~K}$. The decrease in the melting temperature of water can be also related to the confined geometry, that is, nanopores. For example, the melting point of water in hydrophilic silica pores (diameter $\approx 1.5 \mathrm{~nm}$ ) drops by $60^{\circ} .{ }^{34}$ Freezing free water, which would have melted at $273^{\circ}$, was not detected at any of these hydration levels. These results 


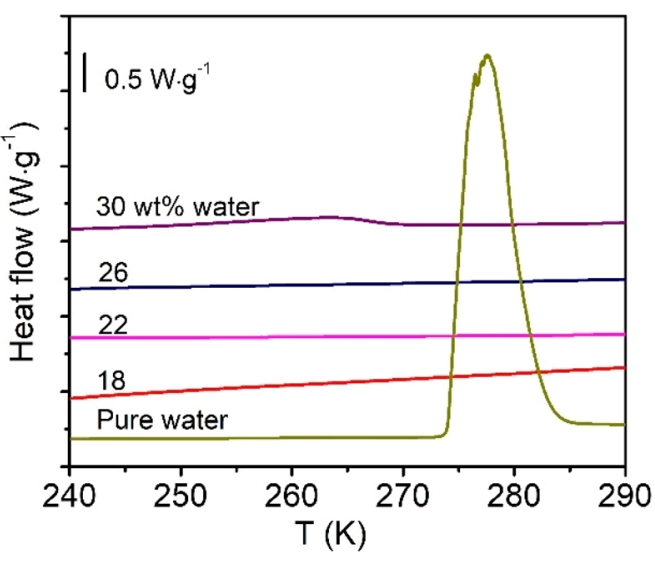

a)

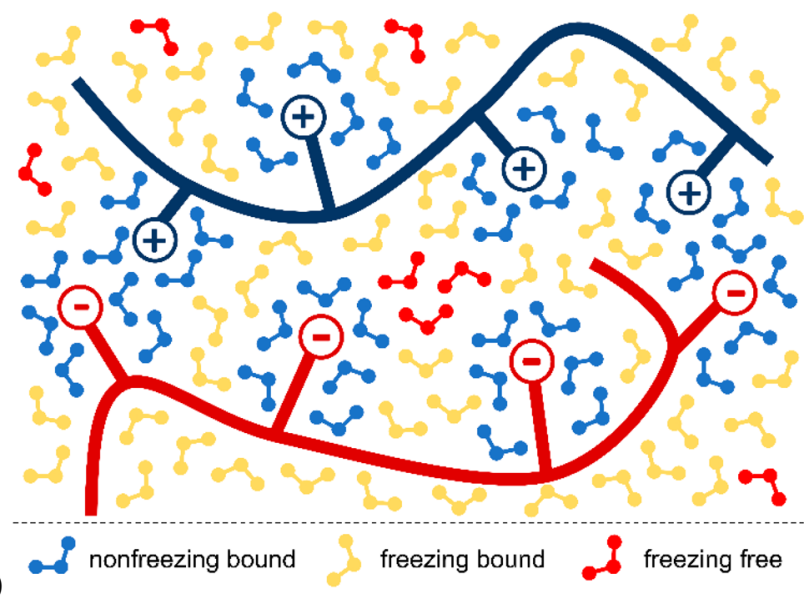

Figure 2. (a) DSC heat flow curves for PDADMA-PSS hydrated PECs with varying water content and pure water to investigate the melting and the states of water in the PEC. First heating is shown at $5 \mathrm{~K} \mathrm{~min}^{-1}$, and the data presented correspond to the "exotherm down" format. (b) An illustration depicting nonfreezing bound water, freezing bound water, and freezing free water.

show that most of the water in hydrated PECs, in the presently studied hydration contents, is tightly bound nonfreezing water.

The glass transition temperature $\left(T_{\mathrm{g}}\right)$ of the hydrated PEC with varying water content, has been recently determined using MDSC $^{24}$ and is remeasured and plotted in Figure S1. The $T_{\mathrm{g}}$ corresponds to the inflection point in the second scan of the sigmoidal reversing heat flow response. We observed that $T_{\mathrm{g}}$ decreased from 384 to $318 \mathrm{~K}$ with increasing hydration. The decrease in $T_{\mathrm{g}}$ with increasing hydration for these PDADMAPSS PECs, as well as for PAH-PAA PECs, has been reported in literature. ${ }^{24,25}$ Recent studies ${ }^{24}$ have shown that the molar ratio of water to intrinsic ion pair was found to be a universal parameter controlling the $T_{\mathrm{g}}$ in both PDADMA-PSS and PAH-PAA PECs.

Accurate determination of the glass transition temperature via simulations is influenced by the local character of this phenomenon: the response depends on the specific local simulation configuration. ${ }^{21}$ On the other hand, the experiments show the average over a huge number of local events over a wide temperature range, $\pm 15 \mathrm{~K}$, see Figure $S 1$. In ref 22 , the glass transition was visible via simulations in PDADMA/PSS assemblies of $4 \mathrm{PDADMA}_{25}-4 \mathrm{PSS}_{25}$ composition at $18 \mathrm{wt} \%$ water as a sudden increase in water mobility and decrease in the mean lifetime of the hydrogen bonds between PSS sulfonate groups and water. However, the simulations presented here correspond to a much larger simulation system, which means the transition here is averaged over a higher number of intrinsic ion pairs and consequently is relatively weak.

Water Distribution in Simulated PEC. To gain more insight into water behavior in hydrated PECs, we examined PE assemblies of same composition, as determined by NAA, ${ }^{24}$ at the same $18,22,26$, and $30 \mathrm{wt} \%$ water content also by $\mathrm{MD}$ simulations. In Figure 3, the water network for MD simulations modeled PECs with 18 and 30 wt \% water content are presented to visualize the distribution of water in the PE assemblies. The figure shows a relatively uniform distribution; that is, no extended water droplets are present. This reflects the PEC structure; see SI for the initial configuration preparation procedure in Table S1 and Figure S2. For the 18 wt \% water system, separated small water clusters are visible, and at the higher hydration, the water network becomes connected. Even then, no large droplets form at $30 \mathrm{wt} \%$ water. This suggests that PE-water binding is stronger than water-water binding. This observation is in line with prior hydrogen bond lifetime observations in PDADMA/PSS PECs that indicate that the PSS-water hydrogen bonds persist twice as long as waterwater bonds. $^{22}$

The corresponding solvent accessible surface area of the PE chains per water molecule, $A_{\mathrm{S}}$, at the different hydration weight percent is presented in Figure 4. The calculation is based on fitting a probe of radius $0.14 \mathrm{~nm}$ into the pores of the PE configurations, see Materials and Methods for details. The systematic (a)

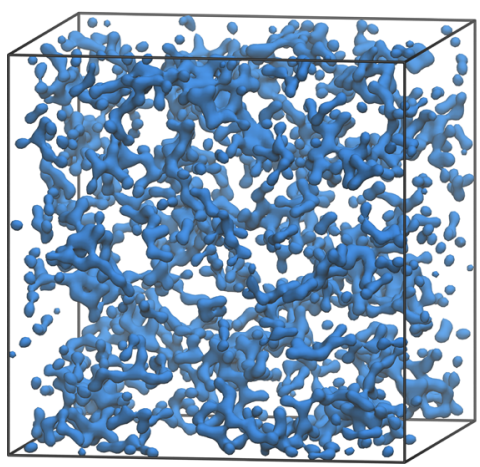

(b)

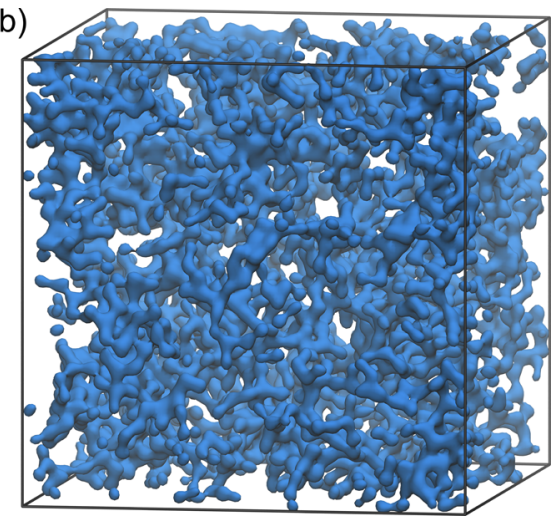

Figure 3. Water network in PEC with (a) 18 wt \% and (b) 30 wt \% water at $290 \mathrm{~K}$. The figures are to scale; increase of water content swells the PEC. Only water (in blue) is shown for clarity. 


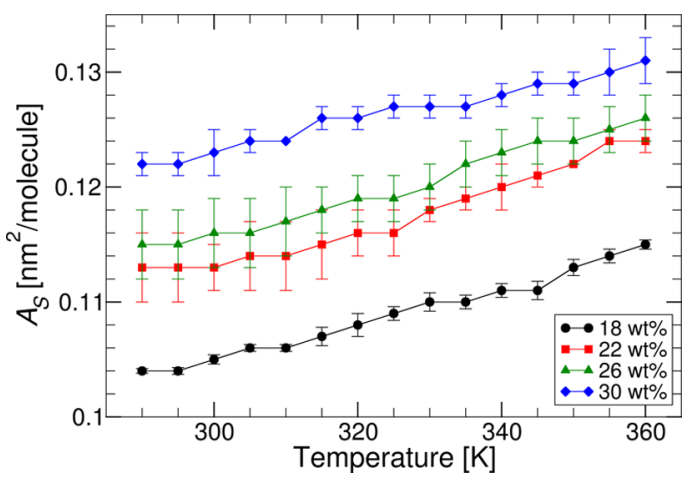

Figure 4. Solvent accessible surface area per water molecule $A_{\mathrm{S}}$ for PECs with different water wt \%.

increase of $A_{\mathrm{S}}$ with the water content means that with increasing hydration, more PE surface becomes available to water, as suggested by the visual observations based on Figure 3. Prior work indicates that the increased chain hydration results in higher mobility of the PE chains. ${ }^{21}$ Increase in PE chain mobility with increasing water content can be expected to decrease the glass transition temperature. Indeed, our MDSC measurements confirm this as the glass transition temperature, $T_{\mathrm{g}}$, for PECs is $384 \mathrm{~K}$ (18 wt \% water content), $355 \mathrm{~K}$ (22 wt \%), $335 \mathrm{~K}(26 \mathrm{wt} \%)$, and $318 \mathrm{~K}$ (30 wt \%) with the respective water contents in parentheses, see Figure $2 \mathrm{~b}$ and Table 1.

Table 1. Nonfreezing Water, $W_{\text {nf }}$, freezing water, $W_{\mathrm{f}}$, and the glass transition temperature, $T_{\mathrm{g}}$, of PDADMA/PSS PECs ${ }^{a}$

$\begin{array}{cccc}W_{\mathrm{c}}[\mathrm{wt} \%] & W_{\mathrm{nf}}[\mathrm{wt} \%] & W_{\mathrm{f}}^{b}[\mathrm{wt} \%] & T_{\mathrm{g}}^{c}[\mathrm{~K}] \\ 18 & 18 & 0 & 384 \\ 22 & 22 & 0 & 355 \\ 26 & 26 & 0 & 335 \\ 30 & 29.1 & 0.9 & 318\end{array}$

${ }^{a}$ Weight percent based on the mass of the hydrated PEC. ${ }^{b}$ Only freezing bound water was observed. Freezing free water was not observed. ${ }^{c}$ Data was adapted from ref 24 .

Figure 4 shows that $A_{\mathrm{S}}$ increases with temperature and that the increase is linear for all systems. Furthermore, the observed slope is independent of the PEC hydration in the studied range. This suggests, that the increase of the PE surface accessed by the solvent is propelled by the increase of the kinetic energy of water molecules with temperature: at higher $T$, the water molecules hydrate regions of the $\mathrm{PE}$ chain that are inaccessible at lower temperatures.

Diffusion versus Temperature. To capture more detail regarding the outcome of the kinetic energy increase of water molecules with temperature, we turn to look at the diffusion behavior. Figure 5 presents the calculated diffusion coefficient, $D$, as well as its translational, $D_{\mathrm{T}}$, and rotational, $D_{\mathrm{R}}$, components for the water molecules hydrating the PECs as a function of the temperature and hydration. The translational and rotational components are derived from the division of the motion spectra. ${ }^{73}$ The data presented in Figure 5 shows that, in agreement with intuition, both diffusion components increase with the temperature. However, the trends are rather different. The translational diffusion, $D_{\mathrm{T}}$, shows a significant dependency on the water content of the system: for the $18 \mathrm{wt} \%$ water system, $D_{\mathrm{T}}$ increases close to linearly with $T$, and the observed changes are relatively small, but for the 30 wt $\%$ water system, $D_{\mathrm{T}}$ shows a more exponential-like growth with $T$. The latter response results in a significantly larger difference in water mobility over the same temperature interval.

For the translational diffusion coefficient, the observed exponential relationship with temperature resembles the Arrhenius equation

$$
D_{\mathrm{T}}=D_{0} \exp \left(-E_{\mathrm{A}} / k_{\mathrm{B}} T\right)
$$

where the parameter $E_{\mathrm{A}}$ is an activation energy for the diffusion and $D_{0}$ a maximal translational diffusion coefficient. A fit of eq 3 to the data of Figure 5 enables extracting these parameters via least-squares fitting. The fits are visible in Figure 5 and the corresponding $E_{\mathrm{A}}$ and $D_{0}$ values are provided in Table S2. The data show that $D_{0}$ increases monotonically with the water content of the PEC. On the other hand, the diffusion activation energy, $E_{A}$, decreases, except for the system with the highest water content. The activation energy represents here the characteristic energy required for breaking the energy barriers in the PEC network, such as PE-water hydrogen bond energy, ${ }^{24}$ and transferring of those water molecules. Hence, $E_{\mathrm{A}}$ composed of enthalpic and entropic components, is an energy related only to the translational molecular motion of water. Its value compared to bulk water is lower than the one measured experimentally based on the self-diffusion coefficient. ${ }^{75}$ For a comparison, corresponding $E_{\mathrm{A}}$ values for hydrogels vary between 16.7 and $62.8 \mathrm{~kJ} / \mathrm{mol}^{76}$ depending on hydration; gels with higher water content have lower activation energy. The activation energy, $E_{\mathrm{A}}$, differs from the van't Hoff enthalpy, which for

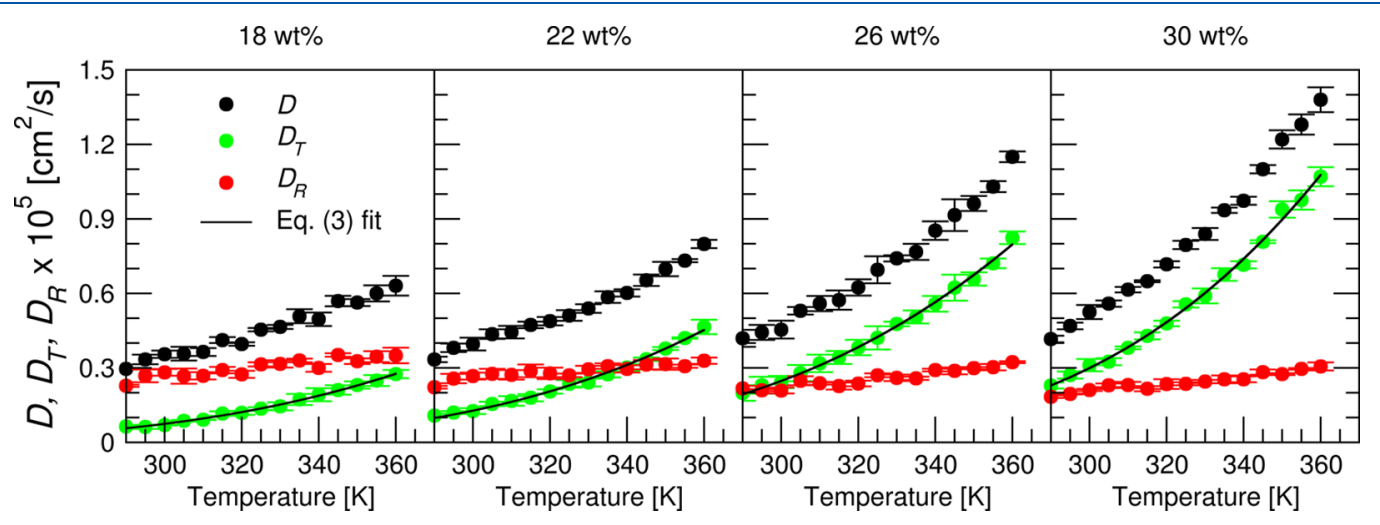

Figure 5. Translational, $D_{\mathrm{T}}$, rotational, $D_{\mathrm{R}}$, and total, $D$, diffusion coefficients of water molecules in the PECs hydrated at different water wt $\%$. The solid lines are fits to eq 3 . 
these systems is associated with the disruption of a single hydrogen bond and, therefore, is independent of the PE type and hydration level. ${ }^{24}$

Contrary to the translational diffusion temperature dependency, the PEC water content does not have significant impact on the temperature response of the rotational diffusion of water molecules, $D_{\mathrm{R}}(T)$; the $D_{\mathrm{R}}(T)$ curves seem to just shift to a higher value with increasing hydration in Figure 5. Bulk water rotational diffusion, however, increases significantly more with temperature.

To elaborate on the response, Figure 6 shows the normalized rotational $D_{\mathrm{R}}^{*}=D_{\mathrm{R}} / D_{\mathrm{R}}^{\mathrm{b}}$ and translational $D_{\mathrm{T}}^{*}=D_{\mathrm{T}} / D_{\mathrm{T}}{ }^{\mathrm{b}}$

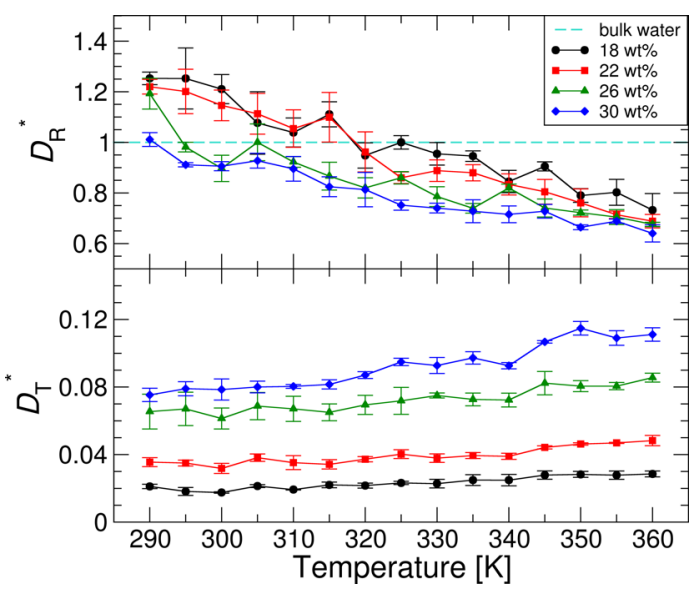

Figure 6. Normalized rotational, $D_{\mathrm{R}}^{*}$, and translational, $D_{\mathrm{T}}^{*}$, diffusion coefficients in the hydrated PECs at different water wt \%. Diffusion coefficients are normalized by the corresponding bulk water rotational or translational diffusion coefficient at the same temperature.

diffusion coefficients of water molecules in PECs, where $D_{\mathrm{R}}{ }^{\mathrm{b}}$ and $D_{\mathrm{T}}{ }^{\mathrm{b}}$ are, respectively, the rotational and translational diffusion coefficients of bulk water at each temperature. The increase of the rotational diffusion of water molecules in bulk water causes $D_{\mathrm{R}}^{*}$ to decrease with temperature, Figure 6 . The observation suggests that a gradual change in the $D_{\mathrm{R}}(T)$ slope with temperature can be expected for a higher water content than examined here.

Diffusion versus Hydration. In Figure 5, the translational diffusion coefficient of water, $D_{\mathrm{T}}$, increases with the PEC water content, but at all water contents, the value remains significantly below bulk water diffusion. However, an opposite trend can be observed for $D_{\mathrm{R}}: D_{\mathrm{R}}$ decreases with increasing water content. Moreover, at low water content and low temperatures, the rotational diffusion of water exceeds that of the bulk water, see Figure 6 . This is related to the confined geometry of the pores in the PECs: as the translational diffusion is hindered strongly by the confinement, a significant part of the molecule energy transfers to the rotational motions.

Comparison of the translational and rotational parts of the total diffusion coefficient of water molecules, Figure 5 reveals that for a PEC with $18 \mathrm{wt} \%$ water, rotational diffusion is higher than translational diffusion through the entire studied temperature range. For the systems with somewhat higher hydration, 22 and 26 wt $\%, D_{\mathrm{T}}$ exceeds $D_{\mathrm{R}}$ around 335 and $295 \mathrm{~K}$, respectively. At $30 \mathrm{wt} \%$ water content, similar to bulk water, the translational diffusion of water in the PEC dominates over the rotational component throughout the examined temperature range. The observed transition from rotational diffusion to translational diffusion dominating regime with an increase of either the hydration or temperature suggests that two different types of water are present in the PEC. One corresponds to water tightly bound to the PE. These water molecules have a relatively small $D_{\mathrm{T}}$ and high $D_{\mathrm{R}}$. The second water species is not a part of PE first hydration shell (is less tightly bound with the PE) and is hence more mobile (higher $D_{\mathrm{T}}$ ). In the following, these two species are referred to as bound and nonbound water but it is important to discern that even for the nonbound water, diffusion is heavily constrained in comparison to bulk water.

Diffusion of Individual Water Molecules. Figure 7 shows the population frequency histograms showing the distribution of individual water molecule total diffusion coefficients in the PECs at different hydration levels, as well as, in bulk water. The data enables gaining more insight toward the bound and nonbound water, and the distribution of water diffusion coefficients in the PEC systems. For bulk water, the diffusion coefficient distribution at different temperatures resembles the Maxwell-Boltzmann distribution. Furthermore, the mean values of $D$ in bulk water, the vertical lines in Figure 7 , are in good agreement with the values calculated for much longer simulations.

The diffusion coefficient distribution of the water molecules in PECs is completely different from the bulk water diffusion coefficient distribution. For the sake of clarity, the $y$-axis in Figure 7 is in linear scale (graph with logarithmic scale is available in the SI, Figure S3) and the $x$-axis ranges differ between the water molecules in PECs and those in bulk water. The data shows that regardless of the temperature and hydration, a vast majority of water molecules have a very low $D$. Such "frozen" molecules can be considered as tightly bound to the PE and constitute most of the water even at the highest $30 \mathrm{wt} \%$ water content PECs. Similar tightly bound water is present in, for example, reverse micelles. ${ }^{77,78}$

The average diffusion coefficients calculated over the individual water molecule diffusion coefficients presented in Figure 7 (20 ps simulation) match the diffusion coefficients calculated using 14 ns averaging time. However, the data shows that only a tiny fraction of the water molecules have a diffusion coefficient close to the average, see Figure 7. For a few selected samples, we calculated the rotational and translational diffusion contributions of water molecules in the different $D$ distribution fractions: the slowest tenth of the molecules had rotational diffusion, $D_{\mathrm{R}}$, contributing to around $90 \%$ of their total $D$ while the fastest tenth had a $90 \%$ of translational contributions $D_{\mathrm{T}}$ in their total $D$. This directly explains the switch from $D_{\mathrm{R}}$ to $D_{\mathrm{T}}$ as the dominant part of diffusion with the temperature and hydration by relating the switch to the fraction of the bound and nonbound water molecules in the system.

The fraction of water with extremely low $D$ can be identified also experimentally via DSC: it corresponds to the nonfreezing water in DSC characterization. For hydrated PECs studied using DSC, only nonfreezing water was observed up to $26 \mathrm{wt} \%$ water content in the PECs and at higher water contents a small amount of freezing water emerged, see Figure 2a). On the other hand, in our simulations, a small fraction of mobile water is present even at the lowest hydration, that is, 18 wt $\%$. The presence of this small amount of mobile water in the simulations could be explained by the difference in examination temperatures: the experiments give us information about the water states close to the water melting temperature or below (freezing bound water), while in the simulations temperatures at 

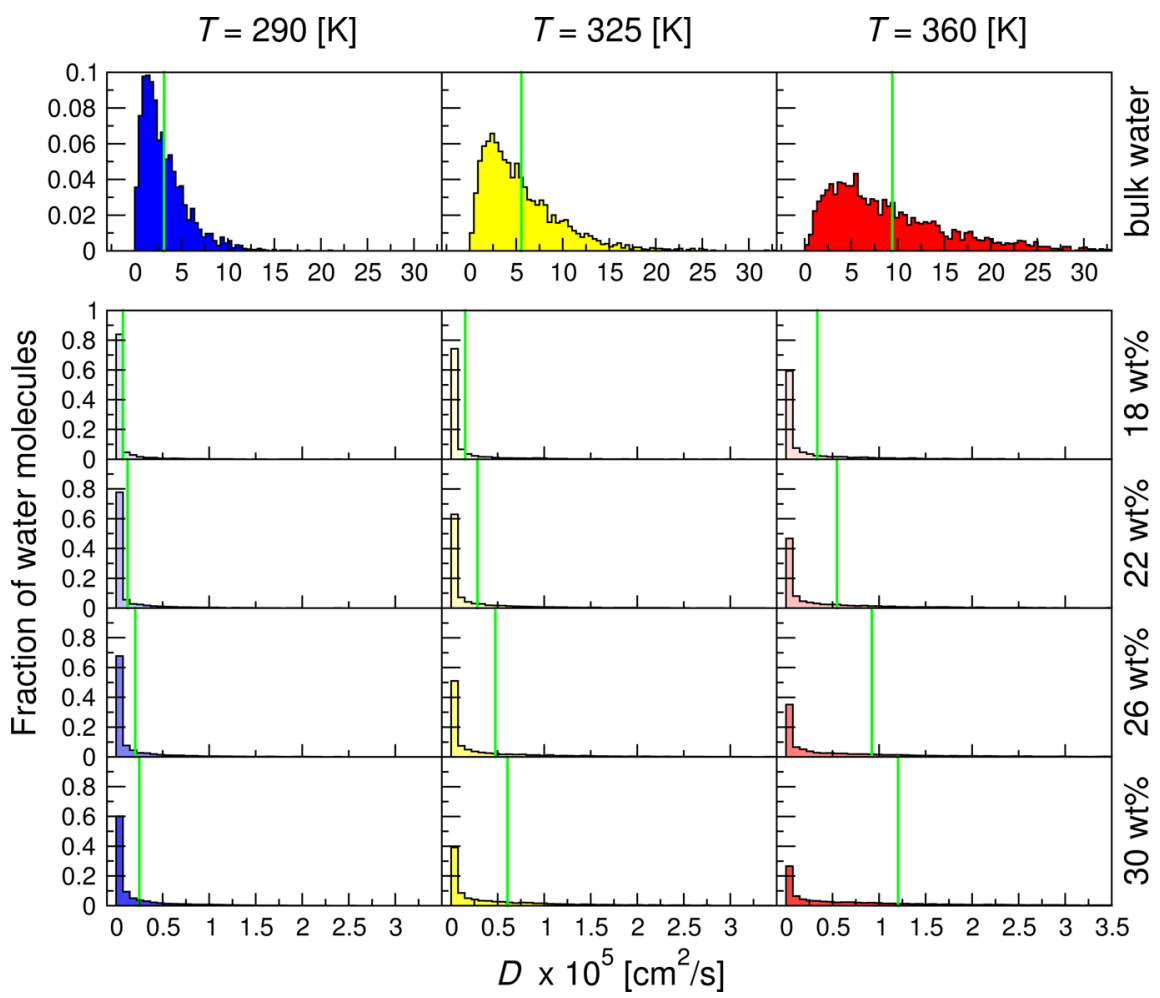

Figure 7. Individual water molecule diffusion coefficients as population frequency histograms for the bulk water and for PECs at different water contents. The data corresponds to the average from three different initial configurations and is calculated over a period of 20 ps. Vertical green lines denote the mean value of the diffusion coefficient. The same data but with a logarithmic axis is provided in the SI (Figure S3). This shows that the majority of water molecules exist in a bound state.

$290 \mathrm{~K}$ and above were investigated. More so, the data of Figure 7 suggests that the distribution of water into bound and nonbound states strongly depends on the temperature. Considering both the experimental and simulation results of this work, for PECs at $<26 \mathrm{wt} \%$ water content at temperatures below $290 \mathrm{~K}$, a complete immobilization of water in the sense of lack of translational diffusion can be expected. On the other hand, for PECs with $30 \mathrm{wt} \%$ water, a small fraction of mobile water remains even at low temperatures, and this water can be frozen, which results in the appearance of a melting peak, Figure 2a).

At 26 and 30 wt \% water content, the amount of bound water exceeds the overall amount of water in the PECs with 18 wt \% water. Regardless, also at $18 \mathrm{wt} \%$ a fraction of more mobile water molecules is present. This suggests that some kind of equilibrium between the bound and nonbound water exists in the PECs. This equilibrium depends also on the temperature. The increase of the amount of bound water with water content seems to have a limiting value toward convergence. This suggests that at some amount of water, the amount of bound water will saturate; further increases in the water amount will increase just the amount of nonbound water. These observations and deductions are very much in agreement with intuition.

The population frequency histograms (Figure 7) for PECs with 18,22 , and $30 \mathrm{wt} \%$ of water at temperatures 290,325 , and $360 \mathrm{~K}$ bear close resemblance to each other. This suggests that temperature and hydration have similar overall effects on the distribution of bound and nonbound water in the PECs and also on the PEC materials response. The equivalent effect of water and temperature on plasticization mechanism was previously reported for poly(vinyl alcohol). ${ }^{27}$ The results here show similar changes in water dynamics within PE assemblies due to increases in either temperature or hydration. The significance of this finding is that the mobility of water molecules is expected to result in a similar plasticization effect in the PEC. This translates to a resulting mechanical response, as observed by Nolte et al. ${ }^{68}$ This finding could provide a bridge between the time-temperature and time-humidity superposition principles proposed for hydrated PE systems in previous studies. ${ }^{79}$

\section{CONCLUSIONS}

The influence of the temperature and hydration on water mobility in hydrated PDADMA/PSS assemblies was investigated theoretically, via all-atom $\mathrm{MD}$ simulations, and experimentally, via MDSC measurements. We explored the temperature and hydration dependency of the binding states of water in PDADMA/PSS complexes. We found that in PECs with hydration up to 26 wt $\%$ water content, only very strongly PE-bound nonfreezing water was present, implying a strong association between water and the polyelectrolytes. For higher hydrations, a small fraction of less tightly bound, freezing bound water was detected as well. The findings might connect with a glass transition temperature decrease with increasing hydration in the PECs and aid to corroborate the crucial role of water as a plasticizer. The MD simulations enabled a quantitative analysis of PE accessible surface area as a function of temperature and hydration: the analysis showed that in the normal hydration range of these $\mathrm{PE}$ assemblies, that is, 18 to $30 \mathrm{wt} \%, \mathrm{PE}-$ water binding dominates over water self-interaction. The diffusion coefficients of water, as well as, its translational and rotational contributions, were calculated. The results demonstrated, that due to the tight binding of the water molecules with the PEs and the confined geometry of the water pores in the PECs, at 
low hydration and temperature, the dominating contributor to total diffusion was the rotational motions whereas at higher hydration and temperature, translational motions became activated; actually, at low hydration and temperature, the rotations were found to exceed in magnitude those in bulk water. The water motions respond similarly to increase in hydration and temperature, that is, effectively similar response can be obtained by either variable.

Investigation of the individual water molecule diffusion revealed that the distribution of water into the bound and nonbound states depends strongly on the hydration and temperature. Both factors seem to change the water distribution in very similar manner, which is in line with the MDSC results suggesting the crucial role of both temperature and water amount in the plasticization of the hydrated PECs. In agreement with the experimental findings, the vast majority of the water molecules hydrating the PECs were immobilized, due to the strong PEwater interactions. The fraction of immobilized water molecules, however, decreases with the increasing temperature and hydration, simultaneously increasing the amount of mobile water in the system, responsible for providing a lubrication effect. In total, the findings highlight the role of hydration as a control parameter in PEC materials properties, raise attention to the similarity of water and temperature as tuning parameters of these materials, and provide an explanation for the similarity.

\section{ASSOCIATED CONTENT}

\section{S Supporting Information}

The Supporting Information is available free of charge on the ACS Publications website at DOI: 10.1021/acs.macromol.8b01441.

Summary of simulation system setup and molecular dynamics steps, fitting parameters of eq 3, MDSC thermograms of hydrated PECs, volume of the simulated systems, and population frequency histograms of individual water molecule diffusion coefficients with logarithmic $y$-axis (PDF)

\section{AUTHOR INFORMATION}

\section{Corresponding Authors}

*E-mail: jodie.lutkenhaus@tamu.edu.

*E-mail: maria.sammalkorpi@aalto.fi.

\section{ORCID $\odot$}

Piotr Batys: 0000-0002-2264-3053

Yanpu Zhang: 0000-0002-8555-6539

Jodie L. Lutkenhaus: 0000-0002-2613-6016

Maria Sammalkorpi: 0000-0002-9248-430X

\section{Notes}

The authors declare no competing financial interest.

\section{ACKNOWLEDGMENTS}

The authors acknowledge the financial support of the Academy of Finland, Grant No. 309324 (M.S.), and National Science Foundation under Grant No. 1609696 (J.L.L). The authors gratefully acknowledge CSC - IT Center for Science, Finland, and RAMI - RawMatTERS Finland Infrastructure for computational resources.

\section{REFERENCES}

(1) Fu, J.; Schlenoff, J. B. Driving Forces for Oppositely Charged Polyion Association in Aqueous Solutions: Enthalpic, Entropic, but Not Electrostatic. J. Am. Chem. Soc. 2016, 138 (3), 980-990.
(2) Das, B. P.; Tsianou, M. From Polyelectrolyte Complexes to Polyelectrolyte Multilayers: Electrostatic Assembly, Nanostructure, Dynamics, and Functional Properties. Adv. Colloid Interface Sci. 2017, 244, 71-89.

(3) Il'ina, A. V.; Varlamov, V. P. Chitosan-Based Polyelectrolyte Complexes: A Review. Appl. Biochem. Microbiol. 2005, 41, 5-11.

(4) Hamman, J. H. Chitosan Based Polyelectrolyte Complexes as Potential Carrier Materials in Drug Delivery Systems. Mar. Drugs 2010, 8, 1305-1322.

(5) Xu, Y.; Mazzawi, M.; Chen, K.; Sun, L.; Dubin, P. L. Protein Purification by Polyelectrolyte Coacervation: Influence of Protein Charge Anisotropy on Selectivity. Biomacromolecules 2011, 12 (5), $1512-1522$.

(6) Roy, K.; Mao, H. Q.; Huang, S. K.; Leong, K. W. Oral Gene Delivery with Chitosan-DNA Nanoparticles Generates Immunologic Protection in a Murine Model of Peanut Allergy. Nat. Med. 1999, 5 (4), 387-391.

(7) Zhang, Y.; Yildirim, E.; Antila, H. S.; Valenzuela, L. D.; Sammalkorpi, M.; Lutkenhaus, J. L. The Influence of Ionic Strength and Mixing Ratio on the Colloidal Stability of PDAC/PSS Polyelectrolyte Complexes. Soft Matter 2015, 11 (37), 7392-7401.

(8) Sui, Z.; Jaber, J. A.; Schlenoff, J. B. Polyelectrolyte Complexes with PH-Tunable Solubility. Macromolecules 2006, 39 (23), 81458152.

(9) Schlenoff, J. B.; Rmaile, A. H.; Bucur, C. B. Hydration Contributions to Association in Polyelectrolyte Multilayers and Complexes: Visualizing Hydrophobicity. J. Am. Chem. Soc. 2008, 130 (41), 13589-13597.

(10) Wang, Z.; Cai, N.; Dai, Q.; Li, C.; Hou, D.; Luo, X.; Xue, Y.; $\mathrm{Yu}, \mathrm{F}$. Effect of Thermal Annealing on Mechanical Properties of Polyelectrolyte Complex Nanofiber Membranes. Fibers Polym. 2014, 15 (7), 1406-1413.

(11) Reid, D. K.; Summers, A.; O’Neal, J.; Kavarthapu, A. V.; Lutkenhaus, J. L. Swelling and Thermal Transitions of Polyelectrolyte Multilayers in the Presence of Divalent Ions. Macromolecules 2016, 49 (16), 5921-5930.

(12) O'Neal, J. T.; Dai, E. Y.; Zhang, Y.; Clark, K. B.; Wilcox, K. G.; George, I. M.; Ramasamy, N. E.; Enriquez, D.; Batys, P.; Sammalkorpi, M.; et al.et al. QCM-D Investigation of Swelling Behavior of Layer-by-Layer Thin Films upon Exposure to Monovalent Ions. Langmuir 2018, 34, 999.

(13) Shiu, C. C.; Wang, S.; Chang, C. H.; Jan, J. S. Poly(1 -Glutamic Acid)-Decorated Hybrid Colloidal Particles from Complex ParticleTemplated Silica Mineralization. J. Phys. Chem. B 2013, 117 (34), 10007-10016.

(14) Kremer, T.; Kovačević, D.; Salopek, J.; Požar, J. Conditions Leading to Polyelectrolyte Complex Overcharging in Solution: Complexation of Poly(Acrylate) Anion with Poly(Allylammonium) Cation. Macromolecules 2016, 49 (22), 8672-8685.

(15) Laaser, J. E.; Lohmann, E.; Jiang, Y.; Reineke, T. M.; Lodge, T. P. Architecture-Dependent Stabilization of Polyelectrolyte Complexes between Polyanions and Cationic Triblock Terpolymer Micelles. Macromolecules 2016, 49 (17), 6644-6654.

(16) Perry, S. L.; Li, Y.; Priftis, D.; Leon, L.; Tirrell, M. The Effect of Salt on the Complex Coacervation of Vinyl Polyelectrolytes. Polymers (Basel, Switz.) 2014, 6 (6), 1756-1772.

(17) Imre, A. W.; Schönhoff, M.; Cramer, C. Unconventional Scaling of Electrical Conductivity Spectra for PSS-PDADMAC Polyelectrolyte Complexes. Phys. Rev. Lett. 2009, 102 (25), No. 255901.

(18) De, S.; Cramer, C.; Schönhoff, M. Humidity Dependence of the Ionic Conductivity of Polyelectrolyte Complexes. Macromolecules 2011, 44 (22), 8936-8943.

(19) Shamoun, R. F.; Hariri, H. H.; Ghostine, R. A.; Schlenoff, J. B. Thermal Transformations in Extruded Saloplastic Polyelectrolyte Complexes. Macromolecules 2012, 45 (24), 9759-9767.

(20) Vidyasagar, A.; Sung, C.; Gamble, R.; Lutkenhaus, J. L. Thermal Transitions in Dry and Hydrated Layer-by-Layer Assemblies 
Exhibiting Linear and Exponential Growth. ACS Nano 2012, 6 (7), 6174-6184.

(21) Zhang, R.; Zhang, Y.; Antila, H. S.; Lutkenhaus, J. L.; Sammalkorpi, M. Role of Salt and Water in the Plasticization of PDAC/PSS Polyelectrolyte Assemblies. J. Phys. Chem. B 2017, 121 (1), 322-333.

(22) Yildirim, E.; Zhang, Y.; Lutkenhaus, J. L.; Sammalkorpi, M. Thermal Transitions in Polyelectrolyte Assemblies Occur via a Dehydration Mechanism. ACS Macro Lett. 2015, 4 (9), 1017-1021.

(23) Vidyasagar, A.; Sung, C.; Losensky, K.; Lutkenhaus, J. L. PHDependent Thermal Transitions in Hydrated Layer-by-Layer Assemblies Containing Weak Polyelectrolytes. Macromolecules 2012, 45 (22), 9169-9176.

(24) Zhang, Y.; Batys, P.; O’Neal, J. T.; Li, F.; Sammalkorpi, M.; Lutkenhaus, J. L. J. L. Molecular Origin of the Glass Transition in Polyelectrolyte Assemblies. ACS Cent. Sci. 2018, 4 (5), 638-644.

(25) Zhang, Y.; Li, F.; Valenzuela, L. D.; Sammalkorpi, M.; Lutkenhaus, J. L. Effect of Water on the Thermal Transition Observed in Poly(Allylamine Hydrochloride)-Poly(Acrylic Acid) Complexes. Macromolecules 2016, 49 (19), 7563-7570.

(26) Stuart, M. A. C.; Huck, W. T. S.; Genzer, J.; Müller, M.; Ober, C.; Stamm, M.; Sukhorukov, G. B.; Szleifer, I.; Tsukruk, V. V.; Urban, M.; et al. Emerging Applications of Stimuli-Responsive Polymer Materials. Nat. Mater. 2010, 9 (2), 101-113.

(27) Hodge, R. M.; Bastow, T. J.; Edward, G. H.; Simon, G. P.; Hill, A. J. Free Volume and the Mechanism of Plasticization in WaterSwollen Poly(Vinyl Alcohol). Macromolecules 1996, 29 (25), 81378143.

(28) Madani, M. M.; Miron, R. R.; Granata, R. D. PALS Free Volume Study of Dry and Water Saturated Epoxies. J. Coat. Technol. 1997, 69 (9), 45-54.

(29) Yao, K. D.; Liu, W. G.; Liu, J. The Unique Characteristics of Water in Chitosan-polyether Semi-IPN Hydrogel. J. Appl. Polym. Sci. 1999, 71 (3), 449-453.

(30) Fu, J.; Abbett, R. L.; Fares, H. M.; Schlenoff, J. B. Water and the Glass Transition Temperature in a Polyelectrolyte Complex. ACS Macro Lett. 2017, 6 (10), 1114-1118.

(31) Ricci, M.; Bruni, F.; Gallo, P.; Rovere, M.; Soper, K. Water in Confined Geometries: Experiments and Simulations. J. Phys.: Condens. Matter 2000, 12 (8A), A345-A350.

(32) Gallo, P.; Rovere, M. Anomalous Dynamics of Confined Water at Low Hydration. J. Phys.: Condens. Matter 2003, 15 (45), 76257633.

(33) Sharma, S.; Debenedetti, P. G. Evaporation Rate of Water in Hydrophobic Confinement. Proc. Natl. Acad. Sci. U. S. A. 2012, 109 (12), 4365-4370.

(34) Jähnert, S.; Vaca Chávez, F.; Schaumann, G. E.; Schreiber, A.; Schönhoff, M.; Findenegg, G. H. Melting and Freezing of Water in Cylindrical Silica Nanopores. Phys. Chem. Chem. Phys. 2008, 10 (39), 6039.

(35) Homshaw, L. G. Freezing And Melting Temperature Hysteresis Of Water In Porous Materials: Application To The Study Of Pore Form. J. Soil Sci. 1980, 31 (3), 399-414.

(36) Vaca Chávez, F.; Schönhoff, M. Pore Size Distributions in Polyelectrolyte Multilayers Determined by Nuclear Magnetic Resonance Cryoporometry. J. Chem. Phys. 2007, 126 (10), 104705.

(37) Hariri, H. H.; Lehaf, A. M.; Schlenoff, J. B. Mechanical Properties of Osmotically Stressed Polyelectrolyte Complexes and Multilayers: Water as a Plasticizer. Macromolecules 2012, 45 (23), 9364-9372.

(38) Müller, M.; Kessler, B.; Richter, S. Preparation of Monomodal Polyelectrolyte Complex Nanoparticles of PDADMAC/Poly(Maleic Acid-Alt- $\alpha$-Methylstyrene) by Consecutive Centrifugation. Langmuir 2005, 21 (15), 7044-7051.

(39) Jin, W.; Toutianoush, A.; Tieke, B. Size- and Charge-Selective Transport of Aromatic Compounds across Polyelectrolyte Multilayer Membranes. Appl. Surf. Sci. 2005, 246 (4), 444-450.
(40) Ostrowska-Czubenko, J.; Pierõg, M.; Gierszewska-Druzyńska, M. Water State in Chemically and Physically Crosslinked Chitosan Membranes. J. Appl. Polym. Sci. 2013, 130 (3), 1707-1715.

(41) Antila, H. S.; Härkönen, M.; Sammalkorpi, M. Chemistry Specificity of DNA-polycation Complex Salt Response: A Simulation Study of DNA, Polylysine and Polyethyleneimine. Phys. Chem. Chem. Phys. 2015, 17 (7), 5279-5289.

(42) Patel, A. P.; Jeon, J.; Mather, P. T.; Dobrynin, A. V. Molecular Dynamics Simulations of Multilayer Polyelectrolyte Films: Effect of Electrostatic and Short-Range Interactions. Langmuir 2006, 22 (24), 9994-10002.

(43) Jeon, J.; Dobrynin, A. V. Molecular Dynamics Simulations of Polyelectrolyte-Polyampholyte Complexes. Effect of Solvent Quality and Salt Concentration. J. Phys. Chem. B 2006, 110 (48), 2465224665.

(44) Jeon, J.; Dobrynin, A. V. Monte Carlo Simulations of Polyampholyte-Polyelectrolyte Complexes: Effect of Charge Sequence and Strength of Electrostatic Interactions. Phys. Rev. E: Stat. Phys., Plasmas, Fluids, Relat. Interdiscip. Top. 2003, 67 (6), No. 061803.

(45) Narambuena, C. F. F.; Leiva, E. P. M. P. M.; Chávez-Páez, M.; Pérez, E. Effect of Chain Stiffness on the Morphology of Polyelectrolyte Complexes. A Monte Carlo Simulation Study. Polymer 2010, 51 (14), 3293-3302.

(46) Kłos, J. S.; Sommer, J.-U. Monte Carlo Simulations of Charged Dendrimer-Linear Polyelectrolyte Complexes and Explicit Counterions. J. Chem. Phys. 2011, 134 (20), 204902.

(47) Hayashi, Y.; Ullner, M.; Linse, P. Complex Formation in Solutions of Oppositely Charged Polyelectrolytes at Different Polyion Compositions and Salt Content $\dagger$. J. Phys. Chem. B 2003, 107 (32), 8198-8207.

(48) Qiao, B.; Cerdà, J. J.; Holm, C. Atomistic Study of Surface Effects on Polyelectrolyte Adsorption: Case Study of a Poly(Styrenesulfonate) Monolayer. Macromolecules 2011, 44 (6), 17071718.

(49) Hoda, N.; Larson, R. G. Modeling the Buildup of Exponentially Growing Polyelectrolyte Multilayer Films. J. Phys. Chem. B 2009, 113 (13), 4232-4241.

(50) Vögele, M.; Holm, C.; Smiatek, J. Coarse-Grained Simulations of Polyelectrolyte Complexes: MARTINI Models for Poly(Styrene Sulfonate) and Poly(Diallyldimethylammonium). J. Chem. Phys. 2015, 143 (24), 243151.

(51) Antila, H. S.; Sammalkorpi, M. Polyelectrolyte Decomplexation via Addition of Salt: Charge Correlation Driven Zipper. J. Phys. Chem. B 2014, 118 (11), 3226-3234.

(52) Qiao, B.; Cerdà, J. J.; Holm, C. Poly(Styrenesulfonate)Poly(Diallyldimethylammonium) Mixtures: Toward the Understanding of Polyelectrolyte Complexes and Multilayers via Atomistic Simulations. Macromolecules 2010, 43 (18), 7828-7838.

(53) Lindahl, E.; Hess, B.; van der Spoel, D. GROMACS 3.0: A Package for Molecular Simulation and Trajectory Analysis. J. Mol. Model. 2001, 7, 306-317.

(54) Berendsen, H. J. C.; van der Spoel, D.; van Drunen, R. GROMACS: A Message-Passing Parallel Molecular Dynamics Implementation. Comput. Phys. Commun. 1995, 91 (1-3), 43-56.

(55) Jorgensen, W. L.; Tirado-Rives, J. The OPLS Potential Functions for Proteins. Energy Minimizations for Crystals of Cyclic Peptides and Crambin. J. Am. Chem. Soc. 1988, 110 (6), 1657-1666.

(56) Jorgensen, W. L.; Gao, J. Monte Carlo Simulations of the Hydration of Ammonium and Carboxylate Ions. J. Phys. Chem. 1986, 90 (10), 2174-2182.

(57) Äqvist, J. Ion-Water Interaction Potentials Derived from Free Energy Perturbation Simulations. J. Phys. Chem. 1990, 94 (21), 80218024.

(58) Chandrasekhar, J.; Spellmeyer, D. C.; Jorgensen, W. L. Energy Component Analysis for Dilute Aqueous Solutions of $\mathrm{Li}+, \mathrm{Na}+, \mathrm{F}-$, and Cl- Ions. J. Am. Chem. Soc. 1984, 106 (4), 903-910.

(59) Jorgensen, W. L.; Madura, J. D. Temperature and Size Dependence for Monte Carlo Simulations of TIP4P Water. Mol. Phys. 1985, 56 (6), 1381-1392. 
(60) Essmann, U.; Perera, L.; Berkowitz, M. L.; Darden, T.; Lee, H.; Pedersen, L. G. A Smooth Particle Mesh Ewald Method. J. Chem. Phys. 1995, 103 (19), 8577-8593.

(61) Hess, B.; Bekker, H.; Berendsen, H. J. C.; Fraaije, J. G. E. M. LINCS: A Linear Constraint Solver for Molecular Simulations. J. Comput. Chem. 1997, 18 (12), 1463-1472.

(62) Miyamoto, S.; Kollman, P. A. Settle: An Analytical Version of the SHAKE and RATTLE Algorithm for Rigid Water Models. J. Comput. Chem. 1992, 13 (8), 952-962.

(63) Humphrey, W.; Dalke, A.; Schulten, K. VMD: Visual Molecular Dynamics. J. Mol. Graphics 1996, 14 (1), 33-38.

(64) Bussi, G.; Donadio, D.; Parrinello, M. Canonical Sampling through Velocity Rescaling. J. Chem. Phys. 2007, 126 (1), 014101.

(65) Parrinello, M.; Rahman, A. Polymorphic Transitions in Single Crystals: A New Molecular Dynamics Method. J. Appl. Phys. 1981, 52 (12), 7182 .

(66) Martinez, L.; Andrade, R.; Birgin, E. G.; Martínez, J. M. PACKMOL: A Package for Building Initial Configurations for Molecular Dynamics Simulations. J. Comput. Chem. 2009, 30 (13), 2157-2164.

(67) Fares, H. M.; Schlenoff, J. B. Diffusion of Sites versus Polymers in Polyelectrolyte Complexes and Multilayers. J. Am. Chem. Soc. 2017, 139 (41), 14656-14667.

(68) Nolte, A. J.; Treat, N. D.; Cohen, R. E.; Rubner, M. F. Effect of Relative Humidity on the Young's Modulus of Polyelectrolyte Multilayer Films and Related Nonionic Polymers. Macromolecules 2008, 41 (15), 5793-5798.

(69) De, S.; Ostendorf, A.; Schönhoff, M.; Cramer, C. Ion Conduction and Its Activation in Hydrated Solid Polyelectrolyte Complexes. Polymers (Basel, Switz.) 2017, 9 (12), 550.

(70) Iturri Ramos, J. J.; Stahl, S.; Richter, R. P.; Moya, S. E. Water Content and Buildup of Poly(Diallyldimethylammonium Chloride)/ Poly(Sodium 4-Styrenesulfonate) and Poly(Allylamine Hydrochloride)/Poly(Sodium 4-Styrenesulfonate) Polyelectrolyte Multilayers Studied by an in Situ Combination of a Quartz Crystal Microbalance with Dissipation Monitoring and Spectroscopic Ellipsometry. Macromolecules 2010, 43 (21), 9063-9070.

(71) Eisenhaber, F.; Lijnzaad, P.; Argos, P.; Sander, C.; Scharf, M. The Double Cubic Lattice Method: Efficient Approaches to Numerical Integration of Surface Area and Volume and to Dot Surface Contouring of Molecular Assemblies. J. Comput. Chem. 1995, 16 (3), 273-284.

(72) Lin, S. T.; Blanco, M.; Goddard, W. A. The Two-Phase Model for Calculating Thermodynamic Properties of Liquids from Molecular Dynamics: Validation for the Phase Diagram of Lennard-Jones Fluids. J. Chem. Phys. 2003, 119 (22), 11792-11805.

(73) Lin, S.-T.; Maiti, P. K.; Goddard, W. A. Two-Phase Thermodynamic Model for Efficient and Accurate Absolute Entropy of Water from Molecular Dynamics Simulations. J. Phys. Chem. B 2010, 114 (24), 8191-8198.

(74) Pascal, T. A.; Lin, S.-T.; Goddard, W. A., III Thermodynamics of Liquids: Standard Molar Entropies and Heat Capacities of Common Solvents from 2PT Molecular Dynamics. Phys. Chem. Chem. Phys. 2011, 13 (1), 169-181.

(75) Wang, J. H. Self-Diffusion Coefficients of Water. J. Phys. Chem. 1965, 69 (12), 4412-4412.

(76) Jeffrey, G. A. An Introduction to Hydrogen Bonding; Oxford University Press: New York, 1997.

(77) Vierros, S.; Sammalkorpi, M. Phosphatidylcholine Reverse Micelles on the Wrong Track in Molecular Dynamics Simulations of Phospholipids in an Organic Solvent. J. Chem. Phys. 2015, 142 (9), No. 094902.

(78) Vierros, S.; Sammalkorpi, M. Role of Hydration in Phosphatidylcholine Reverse Micelle Structure and Gelation in Cyclohexane: A Molecular Dynamics Study. Phys. Chem. Chem. Phys. 2015, 17 (22), 14951-14960.

(79) Cramer, C.; De, S.; Schönhoff, M. Time-Humidity-Superposition Principle in Electrical Conductivity Spectra of IonConducting Polymers. Phys. Rev. Lett. 2011, 107 (2), No. 028301. 